Pacific

Journal of

Mathematics

THE LAPLACIAN ON THE SIERPINSKI GASKET VIA THE METHOD OF AVERAGES

Robert S. StRicharTZ

Volume $201 \quad$ No. 1

November 2001 


\title{
THE LAPLACIAN ON THE SIERPINSKI GASKET VIA THE METHOD OF AVERAGES
}

\author{
Robert S. Strichartz
}

\begin{abstract}
We show how the symmetric Laplacian on the Sierpinski gasket, together with its associated Dirichlet form and harmonic functions, can be defined entirely in terms of average values of a function over basic sets. This approach combines the constructive limit-of-difference-quotients method of Kigami and the method of averages introduced by Kusuoka and Zhou for the Sierpinski carpet.
\end{abstract}

\section{Introduction.}

A direct construction of a Laplacian on the Sierpinski gasket as a limit of difference quotients was given by Kigami [Ki1], who later extended the method to a class of self-similar fractals called post critically finite (p.c.f.) $[\mathbf{K i 2}, \mathbf{K i 3}]$. At about the same time, Kusuoka and Zhou $[\mathbf{K Z}]$ introduced what we will call the method of averages for defining a Laplacian on the Sierpinski carpet, a fractal that is not p.c.f. The method of averages uses average values of functions over basic sets rather than pointwise values in defining all operations. In this paper we will show how the method of averages can be used to define the Laplacian on the Sierpinski gasket. (Since there are many different Laplacians obtainable by the method of [Ki2], we should call this the symmetric Laplacian.) It would be nice to be able to use the method for all Laplacians on p.c.f. fractals, but it is not clear at present how to do this. Ultimately, the goal is to use the method of averages to define Laplacians on wider classes of fractals. To advance these goals it is worthwhile to have a basic example worked out in detail. As we will see, the formulas involved are a bit more complicated than the analogous ones for pointwise values. Also, since average values play such an important role in the usual theory of harmonic functions, it is of independent interest to understand the properties of average values of harmonic functions on the Sierpinski gasket.

For the convenience of the reader, we summarize the results from [Ki1] that we will use. In principle we should establish all properties of the theory directly in terms of the average quantities, and then show that the theory is equivalent to the standard pointwise one. However, in the interest of brevity, we will make the connection between average and pointwise quantities at 
the outset, and make use of properties from the pointwise theory whenever convenient to simplify the proofs. A different characterization of the domain of the Dirichlet form, based on integrated quantities, is given in $[\mathbf{J}]$.

\section{Harmonic functions.}

The Sierpinski gasket $K$ is generated by the i.f.s. consisting of 3 mappings in the plane, $F_{j} x=\frac{1}{2} x+\frac{1}{2} q_{j}, j=0,1,2$, where $q_{0}, q_{1}, q_{2}$ are the vertices of an equilateral triangle. We approximate the fractal $K$ by a sequence of graphs $\Gamma_{0}, \Gamma_{1}, \ldots$ with vertices $V_{0} \subseteq V_{1} \subseteq V_{2} \subseteq \cdots$, where $V_{0}=\left\{q_{0}, q_{1}, q_{2}\right\}$ and $V_{k+1}=\bigcup_{j=0}^{2} F_{j} V_{k}$. The edge relation for $\Gamma_{m}$, denoted $x \sim_{m} y$ for $x, y \in V_{m}$ and $x \neq y$, is defined by the existence of a word $w=\left(w_{1}, \ldots, w_{m}\right)$ of length $|w|=m$ such that $x, y \in F_{w} K$, where $F_{w}=F_{w_{1}} \circ F_{w_{2}} \circ \cdots \circ F_{w_{m}}$. The simple graph energy form on $\Gamma_{m}$ is

$$
E_{m}(u, v)=\sum_{x \sim_{m} y}(u(x)-u(y))(v(x)-v(y))
$$

and the renormalized energy $\mathcal{E}_{m}$ is given by

$$
\mathcal{E}_{m}(u, v)=\left(\frac{5}{3}\right)^{m} E_{m}(u, v)
$$

where $u$ and $v$ denote continuous functions on $K$ and, by abuse of notation, their restriction to $V_{m}$.

We regard $V_{0}$ as the boundary of each graph $V_{m}$, and also of $K$. A function $h$ on $V_{m}$ (for $m \geq 1$ ) is called graph harmonic if it satisfies

$$
h(x)=\frac{1}{4} \sum_{y \sim_{m} x} h(y)
$$

for all non-boundary points $x$ (note that such points have exactly 4 neighbors in $V_{m}$ ). It is easy to see that this is equivalent to the property that $h$ minimizes the energy $E_{m}(u, u)$ among all functions $u$ with the same boundary values. The following proposition summarizes the basic results (from [Ki1]) concerning the Dirichlet form and harmonic functions on $K$, and justifies the choice of renormalization factor in (2.2):

Proposition 2.1. For any continuous function $u$ on $K$, the sequence $\mathcal{E}_{m}(u, u)$ is monotone increasing, so

$$
\mathcal{E}(u, u)=\lim _{m \rightarrow \infty} \mathcal{E}_{m}(u, u)
$$

is well-defined in $[0, \infty]$, and $\mathcal{E}(u, u)=0$ if and only if $u$ is constant. Denote by $\operatorname{dom} \mathcal{E}$ the set of continuous functions for which $\mathcal{E}(u, u)<\infty$. Then $\operatorname{dom} \mathcal{E}$ 
modulo constants is a Hilbert space with inner product

$$
\mathcal{E}(u, v)=\lim _{m \rightarrow \infty} \mathcal{E}_{m}(u, v) .
$$

A function $h$ is called harmonic on $K$ if it minimizes energy $\mathcal{E}(u, u)$ among functions with the same boundary values. Then $h$ is harmonic if and only if its restriction to every $V_{m}$ is graph harmonic. For a harmonic function, $\mathcal{E}_{m}(h, h)=\mathcal{E}(h, h)$ for every $m$. The space of harmonic functions is 3-dimensional, with each harmonic function determined uniquely from its boundary values by means of the following harmonic algorithm: If the values of $h$ on $V_{m}$ are known, and the value $h(x)$ for $x \in V_{m+1} \backslash V_{m}$ is desired, find $w$ of length $|w|=m$ such that $x \in F_{w} K$, and set

$$
h(x)=\frac{2}{5} h\left(F_{w} q_{j}\right)+\frac{2}{5} h\left(F_{w} q_{j+1}\right)+\frac{1}{5} h\left(F_{w} q_{j+2}\right)
$$

if $x$ lies between $F_{w} q_{j}$ and $F_{w} q_{j+1}$ (cyclic notation of indices). For any $u \in \operatorname{dom} \mathcal{E}$ the normal derivatives

$$
\partial_{n} u\left(q_{j}\right)=\lim _{m \rightarrow \infty}\left(\frac{5}{3}\right)^{m}\left(2 u\left(q_{j}\right)-u\left(F_{j}^{m} q_{j+1}\right)-u\left(F_{j}^{m} q_{j+2}\right)\right)
$$

exist at boundary points, and for harmonic functions it is not necessary to take the limit in (2.7).

Our first goal is to obtain the same results for quantities defined in terms of average values. Let $\mu$ denote the self-similar probability measure on $K$ satisfying

$$
\left\{\begin{array}{l}
\mu(B)=\frac{1}{3} \mu\left(F_{0}^{-1} B\right)+\frac{1}{3} \mu\left(F_{1}^{-1} B\right)+\frac{1}{3} \mu\left(F_{2}^{-1} B\right) \text { or } \\
\int f d \mu=\frac{1}{3} \sum_{j=0}^{2} \int f \circ F_{j} d \mu .
\end{array}\right.
$$

We define the averages

$$
A_{w}(f)=\int f \circ F_{w} d \mu=3^{-m} \int_{F_{w} K} f d \mu
$$

for any word $w$. If we write $A_{w}(f)=a_{w}$ then we have

$$
3 a_{w}=a_{w 0}+a_{w 1}+a_{w 2}
$$

(here $w 0$ denotes $\left(w_{1}, \ldots, w_{m}, 0\right)$, etc). Conversely, given numbers $a_{w}$ for every $w$, with (2.10) holding, there exists a continuous function $f$ such that $A_{w}(f)=a_{w}$ provided the function $w \rightarrow a_{w}$ satisfies its own form of uniform continuity. To state this condition we first define a graph $\widetilde{\Gamma}_{m}$ whose vertices are the words $w$ of length $|w|=m$, and whose edge relation, also denoted $w \sim_{m} w^{\prime}$, is defined by the condition that $F_{w} K \cap F_{w^{\prime}} K$ is non-empty. The boundary of $\widetilde{\Gamma}_{m}$ is defined to be the 3 constant words, denoted $0^{m}, 1^{m}, 2^{m}$. Figure 2.1 shows $\widetilde{\Gamma}_{1}, \widetilde{\Gamma}_{2}$ and $\widetilde{\Gamma}_{3}$. 

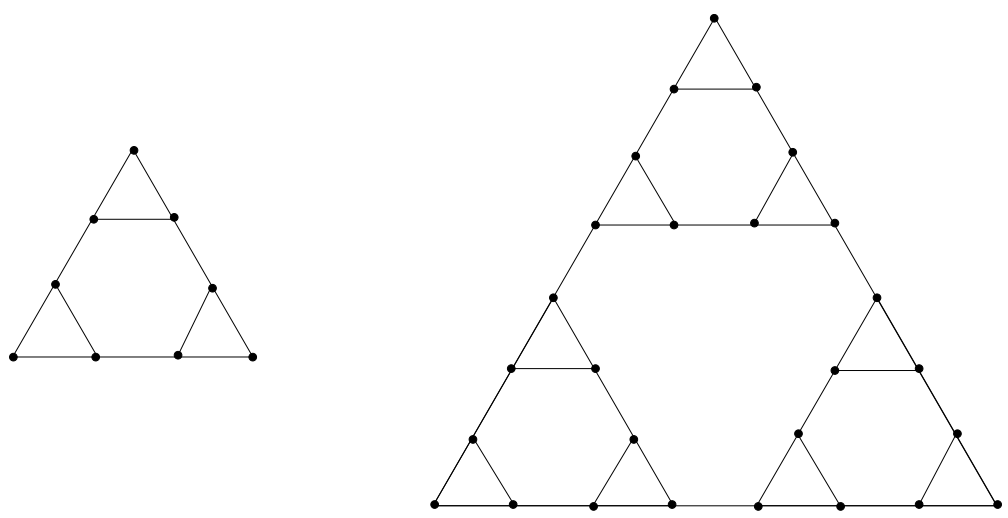

Figure 2.1. a) $\widetilde{\Gamma}_{1}$. b) $\widetilde{\Gamma}_{2}$. c) $\widetilde{\Gamma}_{3}$.

Definition 2.2. A function $w \rightarrow a_{w}$ is uniformly continuous if for every $\varepsilon>0$ there exists $m(\varepsilon)$ such that for all $m \geq m(\varepsilon)$ we have $\left|a_{w}-a_{w^{\prime}}\right| \leq \varepsilon$ provided $|w|=\left|w^{\prime}\right|=m$ and $w \sim_{m} w^{\prime}$.

Lemma 2.3. $a_{w}=A_{w}(f)$ for some continuous function $f$, if and only if (2.10) holds and $w \rightarrow a_{w}$ is uniformly continuous.

We leave the proof as an exercise.

Now we would like to characterize harmonic functions in terms of their average values. If we write the graph harmonic condition for $\widetilde{\Gamma}_{m}(m \geq 2)$ it says

(2.11) $3 a_{w}=\sum_{w^{\prime} \sim_{m} w} a_{w^{\prime}} \quad$ for all non-boundary $\quad w \quad$ of length $|w|=m$.

Note that each such $w$ has exactly 3 neighbors in $\widetilde{\Gamma}_{m}$. The harmonic extension problem (from $\widetilde{\Gamma}_{m}$ to $\widetilde{\Gamma}_{m+1}$ ) can be stated as follows: Given values $a_{w}$ for $|w| \leq m$ satisfying (2.11) for $|w| \leq m$ and (2.10) for $|w| \leq m-1$, define $a_{w}$ for $|w|=m+1$ so that (2.11) holds for $|w|=m+1$ and (2.10) holds for $|w|=m$. The solution will be given by the following harmonic algorithm:

$$
\left\{\begin{array}{l}
a_{w j k}=\frac{4}{5} a_{w j}+\frac{1}{5} a_{w k} \quad j \neq k \\
a_{w j j}=\frac{8}{5} a_{w j}-\frac{3}{5} a_{w}
\end{array}\right.
$$

for $|w|=m-1$.

Lemma 2.4. Given values $a_{w}$ for $|w| \leq m$ satisfying (2.11) for $|w| \leq m$ and (2.10) for $|w| \leq m-1$, define $a_{w}$ for $|w|=m+1$ by (2.12). Then (2.11) holds for $|w|=m+1$ and (2.10) holds for $|w|=m$. 
Proof. First we verify (2.10), namely $3 a_{w j}=a_{w_{j 0}}+a_{w_{j 1}}+a_{w_{j 2}}$, for $|w|=$ $m-1$. For simplicity of notation take $j=0$. Then by $(2.12)$

$$
\begin{aligned}
a_{w 00}+a_{w 01}+a_{w 02} & =\frac{8}{5} a_{w 0}-\frac{3}{5} a_{w}+\frac{4}{5} a_{w 0}+\frac{1}{5} a_{w 1}+\frac{4}{5} a_{w 0}+\frac{1}{5} a_{w 2} \\
& =3 a_{w 0}+\frac{1}{5}\left(a_{w 0}+a_{w 1}+a_{w 2}-3 a_{w}\right)
\end{aligned}
$$

and $a_{w 0}+a_{w 1}+a_{w 2}-3 a_{w}=0$ by (2.10), known to be true since $|w|=m-1$.

Next we verify (2.11). We consider two cases. In the first case the word has the form $w j k$ for $j \neq k$ and $|w|=m-1$. For simplicity of notation take $w_{01}$, with neighbors $w_{10}, w_{00}$ and $w_{02}$. By $(2.12)$

$$
\begin{aligned}
a_{w 10}+a_{w 00}+a_{w 02} & =\frac{4}{5} a_{w 1}+\frac{1}{5} a_{w 0}+\frac{8}{5} a_{w 0}-\frac{3}{5} a_{w}+\frac{4}{5} a_{w 0}+\frac{1}{5} a_{w 2} \\
& =\frac{13}{5} a_{w 0}+\frac{4}{5} a_{w 1}+\frac{1}{5} a_{w 2}-\frac{3}{5} a_{w} .
\end{aligned}
$$

Using (2.10) for $a_{w}$ this becomes $3\left(\frac{4}{5} a_{w 0}+\frac{1}{5} a_{w 1}\right)$, and this is $3 a_{w 01}$ by (2.12). The second case is a word of the form $w j j$ for $|w|=m-1$. For simplicity of notation take $j=0$. Let $w^{\prime}$ denote the word of length $m-1$ that neighbors $w$ on the side of $w 00$, and assume $w^{\prime} 11$ neighbors $w 00$ (the other possibility is $w^{\prime} 22$ ). That means $w 00$ has neighbors $w 01, w 02$ and $w^{\prime} 11$ (see Figure 2.2). Also $w 0$ has neighbors $w 1, w 2$ and $w^{\prime} 1$, and $w^{\prime} 1$ has neighbors $w^{\prime} 0, w^{\prime} 2$ and $w 0$, and this means

$$
\left\{\begin{array}{l}
3 a_{w 0}=a_{w 1}+a_{w 2}+a_{w^{\prime} 1} \text { and } \\
3 a_{w^{\prime} 1}=a_{w 0}+a_{w^{\prime} 0}+a_{w^{\prime} 2}=a_{w 0}+3 a_{w^{\prime}}-a_{w^{\prime} 1}
\end{array}\right.
$$

by (2.11) and (2.10). Now by (2.12) we have

$$
\begin{aligned}
a_{w^{\prime} 11}+a_{w 01}+a_{w 02} & =\frac{8}{5} a_{w^{\prime} 1}-\frac{3}{5} a_{w^{\prime}}+\frac{4}{5} a_{w 0}+\frac{1}{5} a_{w 1}+\frac{4}{5} a_{w 0}+\frac{1}{5} a_{w 2} \\
& =\frac{8}{5} a_{w 0}+\frac{1}{5} a_{w 1}+\frac{1}{5} a_{w 2}+\frac{8}{5} a_{w^{\prime} 1}-\frac{3}{5} a_{w^{\prime}} .
\end{aligned}
$$

Using (2.13) to eliminate $a_{w^{\prime} 1}$ and $a_{w}$ yields $3\left(\frac{7}{5} a_{w 0}-\frac{1}{5} a_{w 1}-\frac{1}{5} a_{w 2}\right)$, which equals $3\left(\frac{8}{5} a_{w 0}-\frac{3}{5} a_{w}\right)$ by $(2.10)$, and this is $3 a_{w 00}$ by $(2.12)$.

If we start with arbitrary values for $a_{0}, a_{1}, a_{2}$, then by repeated use of (2.12) we can define $a_{w}$ for all $w$ so that (2.10) and (2.11) hold. It will be true that $a_{w}$ is uniformly continuous in the sense of Definition 2.2, so that by Lemma 2.3 there is a continuous function $h$ with $A_{w}(h)=a_{w}$, but it is rather tricky to show this directly. Instead we will show that there is a harmonic function $h$ with $A_{w}(h)$ satisfying the same identities as $a_{w}$, so $A_{w}(h)=a_{w}$ indirectly. 

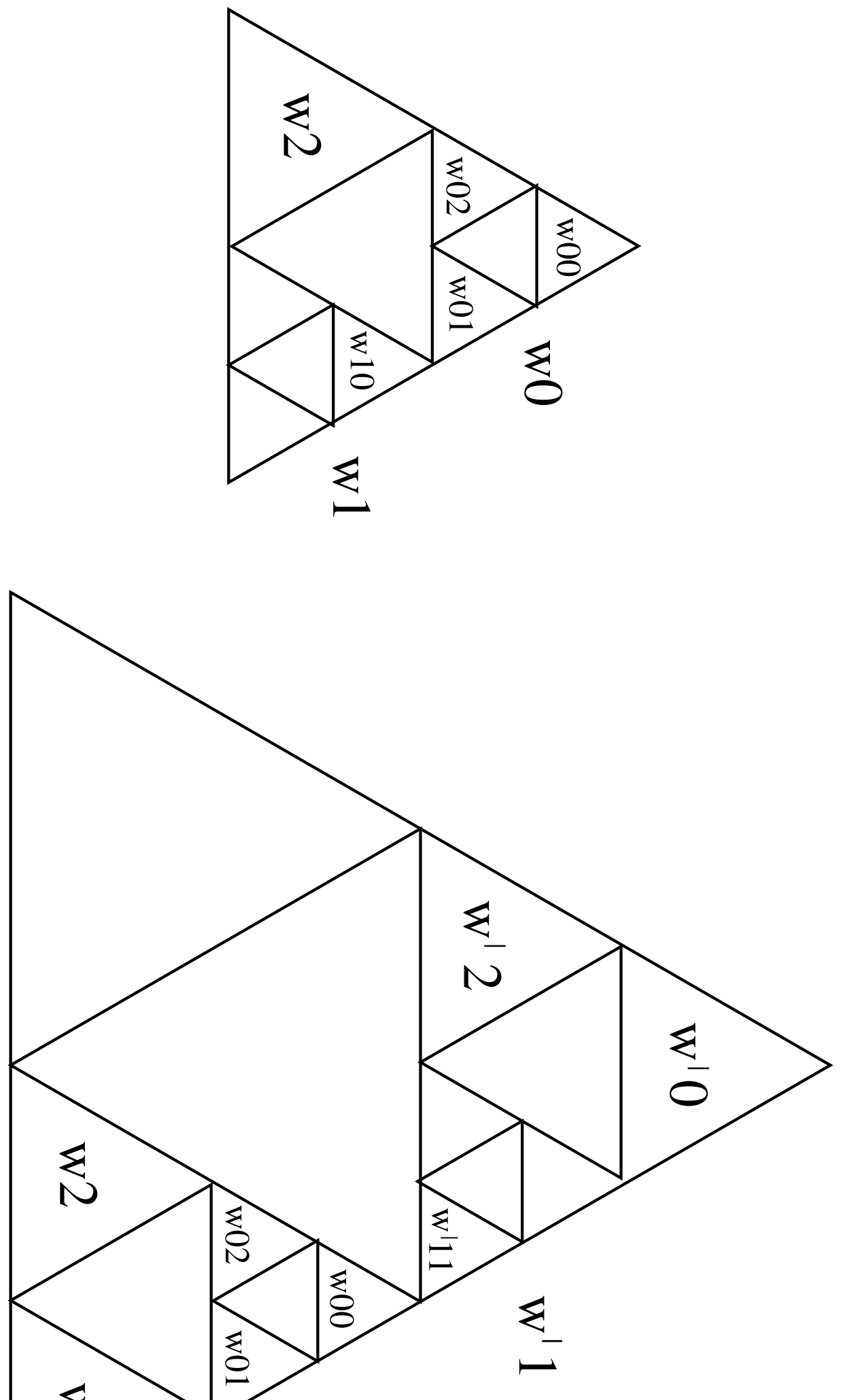
Theorem 2.5. (a) A continuous function $h$ is harmonic if and only if its average values $A_{w}(h)$ satisfy (2.11) for all $m \geq 2$.

(b) If $h$ is harmonic then $A_{w}(h)$ satisfy (2.12).

(c) Given arbitrary values for $a_{0}, a_{1}, a_{2}$, and defining $a_{w}$ for all $w$ via (2.12), there exists a harmonic function $h$ such that $A_{w}(h)=a_{w}$.

(d) The boundary values and normal derivatives of a harmonic function are given in terms of average values by

$$
\left\{\begin{array}{l}
h\left(q_{j}\right)=\frac{5}{2} A_{j^{m+1}}(h)-\frac{3}{2} A_{j^{m}}(h) \quad \text { for any } m \geq 0, \\
\partial_{n} h\left(q_{j}\right)=\frac{15}{2}\left(\frac{5}{3}\right)^{m}\left(A_{j^{m+1}}(h)-A_{j^{m}}(h)\right) \text { for any } m \geq 0 .
\end{array}\right.
$$

Proof. For any harmonic function $h$ we have

$$
\int h d \mu=\frac{1}{3} \sum_{j=0}^{2} h\left(q_{j}\right)
$$

by rotational symmetry, hence

$$
A_{w}(h)=\int h \circ F_{w} d \mu=\frac{1}{3} \sum_{j=0}^{2} h\left(F_{w} q_{j}\right)
$$

since $h \circ F_{w}$ is also harmonic. We can use (2.15) to replace any equation involving average values by one involving pointwise values. In particular, if we do this for (2.11), we obtain a certain identity which is also the average of the graph harmonic identity (2.3) at the 3 vertices $F_{w} q_{j}$ of $F_{w} K$, as can be seen from Figure 2.3. Since (2.3) holds for harmonic functions, so does (2.11), proving one implication in (a).

To prove (b) it suffices to show (2.12) for the empty word $w$ and for the single harmonic function whose values and averages are shown in Figure 2.4. This is an exercise in arithmetic. Similarly, we can verify (2.14) for $m=0,1$ directly for this function. It follows easily from (2.12) that the right sides of (2.14) are independent of $m$, proving (d).

To prove (c) we construct a harmonic function $h$ satisfying $A_{j}(h)=a_{j}$, $j=0,1,2$ simply by setting

$$
\begin{aligned}
& h\left(q_{0}\right)=2 a_{0}-\frac{1}{2} a_{1}-\frac{1}{2} a_{2} \\
& h\left(q_{1}\right)=2 a_{1}-\frac{1}{2} a_{0}-\frac{1}{2} a_{2} \\
& h\left(q_{2}\right)=2 a_{2}-\frac{1}{2} a_{0}-\frac{1}{2} a_{1}
\end{aligned}
$$

in view of (d), and extending $h$ by the harmonic algorithm (2.6). Since both $A_{w}(h)$ and $a_{w}$ satisfy (2.12), it follows that $A_{w}(h)=a_{w}$ for all $w$. 


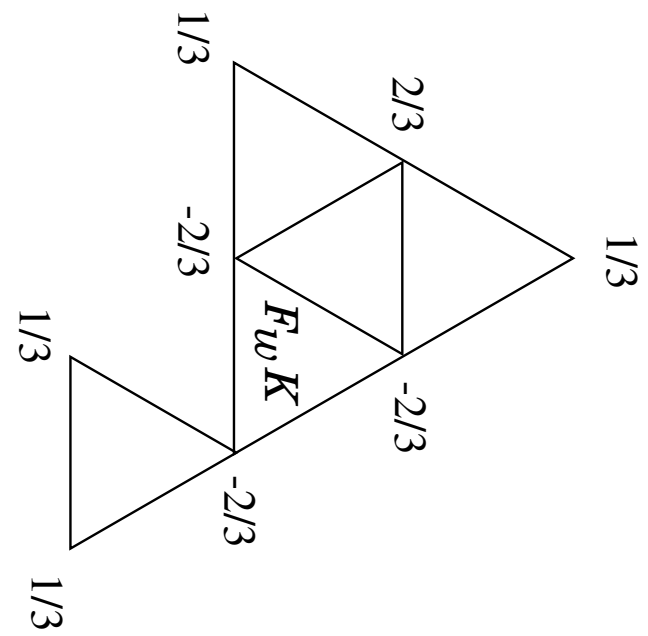

Figure 2.3. The weights at the vertices shown have the property that for any harmonic, the sum of the weight times the value of the function vanishes. These weights were obtained by substituting (2.15) in (2.11). But the same weights may also be obtained by averaging (2.3) at the 3 vertices of $F_{w} K$.

Finally, to complete the proof of (a), assume that $u$ is a continuous function (it is in fact not necessary to assume continuity) such that $a_{w}=A_{w}(u)$ satisfies (2.11). By (c) there exists a harmonic function $h$ with $a_{w}=A_{w}(h)$, hence $A_{w}(u)=A_{w}(h)$ for all $w$. It follows by standard measure theory that $u=h$ a.e., so $u$ is harmonic.

Remarks. (1) It is not true that condition (2.15) characterizes harmonic functions. For a counterexample take the piecewise harmonic function whose values on $V_{1}$ are shown in Figure 2.5. Interestingly, there is an analogous characterization of harmonic functions in Euclidean spaces: A continuous function whose average value on a ball is the same as its average value on the boundary of the ball, for every ball, must be harmonic. (We are grateful to L. Zalcman for this observation, which is obtainable from results in $[\mathbf{Z}]$.) Of course the analogy is not exact between sets $F_{w} K$ in $K$ and balls in Euclidean space.

(2) It is also easy to compute the transverse derivatives defined in [S2] in terms of average values, namely

$$
\partial_{T} h\left(q_{j}\right)=\frac{1}{5^{m}}\left(h\left(F_{j}^{m} q_{j+1}\right)-h\left(F_{j}^{m} q_{j+2}\right)\right)
$$




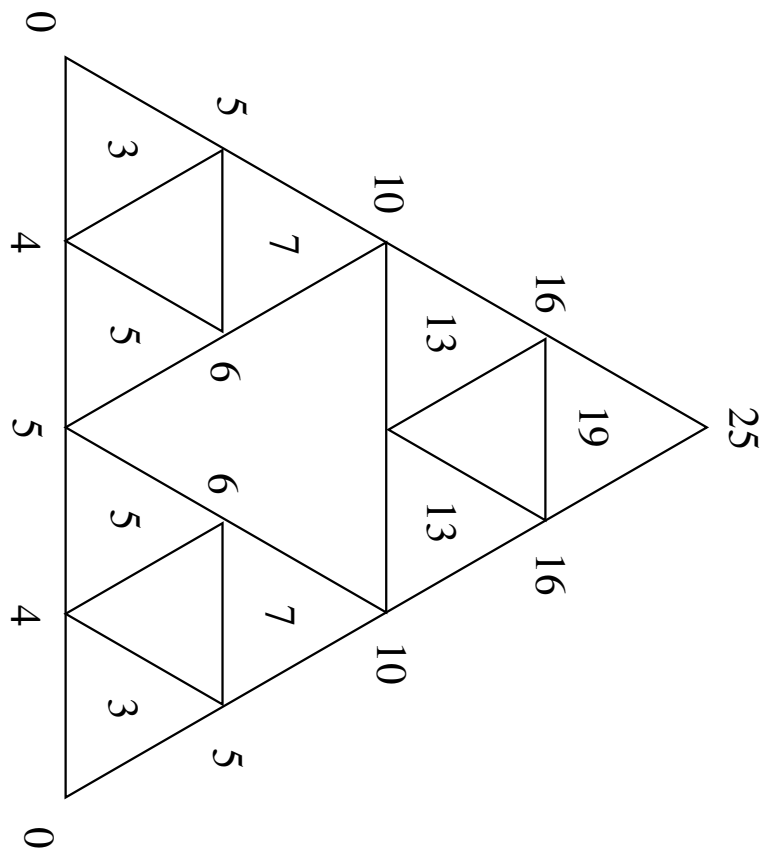

Figure 2.4. The values at vertices and averages (in triangles) for a typical harmonic function.

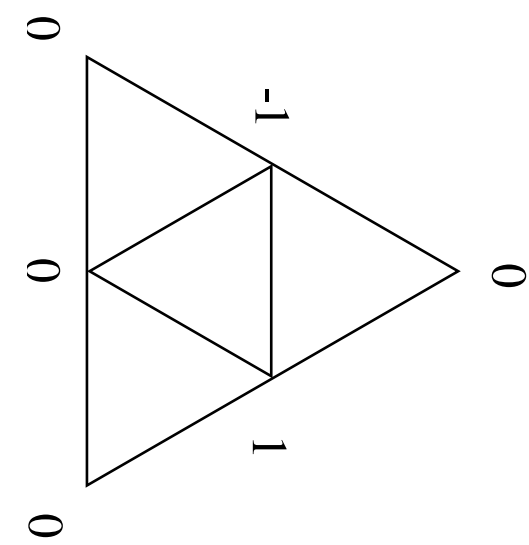

Figure 2.5. The values of a piecewise harmonic function satisfying (2.15) which is not harmonic.

for any $m \geq 0$ is the same as

$$
\left(\frac{5}{2}\right) \frac{1}{5^{m}}\left(A_{j^{m}(j+1)}(h)-A_{j^{m}(j+2)}(h)\right)
$$


for any $m \geq 0$. This can be seen directly for $m=0,1$ for the harmonic function in Figure 2.4. On the other hand the right side of (2.16) is independent of $m$ by (2.6), and the same is true for (2.17) by (2.12).

\section{The Dirichlet form.}

Now we consider the expression of the Dirichlet form in terms of average values. The graph $\widetilde{\Gamma}_{m}$ gives rise to a simple energy form

$$
\widetilde{E}_{m}(u, v)=\sum_{w \sim_{m} w^{\prime}}\left(A_{w}(u)-A_{w^{\prime}}(u)\right)\left(A_{w}(v)-A_{w^{\prime}}(v)\right) .
$$

In analogy with the pointwise formula (2.2) we might guess a renormalization factor of $(5 / 3)^{m}$. In fact we choose

$$
\widetilde{\mathcal{E}}_{m}(u, v)=\frac{3}{2}\left(\left(\frac{3}{5}\right)^{m}-\left(\frac{3}{5}\right)^{2 m}\right)^{-1} \widetilde{E}_{m}(u, v)
$$

to define the renormalized energy. In the limit we obtain the same thing (the $3 / 2$ factor in (3.2) is chosen to obtain the same energy as in the pointwise definition). The reason for the choice of the constant in (3.2) is that for harmonic functions the expression is independent of $m$.

Theorem 3.1. For a harmonic function $h, \widetilde{\mathcal{E}}_{m}(h, h)$ is independent of $m$, and in fact equal to $\mathcal{E}_{m}(h, h)$.

Proof. We will establish the recursion formulas

$$
\left\{\begin{array}{l}
\widetilde{E}_{1}(h, h)=\frac{4}{25} E_{0}(h, h) \\
\widetilde{E}_{m}(h, h)=\frac{4}{25} E_{m-1}(h, h)+\frac{9}{25} \widetilde{E}_{m-1}(h, h) \text { for } m \geq 2 .
\end{array}\right.
$$

Since $E_{m-1}(h, h)=\left(\frac{3}{5}\right)^{m-1} E_{0}(h, h)$ the result follows from (3.3) by a routine argument.

To establish (3.3) we consider two types of adjacent pairs of words of length $m$. The first type is of the form $(w j, w k)$, for $j \neq k$ and $|w|=m-1$. Now from (2.15) and (2.6) we obtain easily

$$
A_{w j}(h)=\frac{2}{5} h\left(F_{w} q_{j}\right)+\frac{3}{5} A_{w}(h) .
$$

Then we have

$$
\begin{aligned}
& \left(A_{w 0}(h)-A_{w 1}(h)\right)^{2}+\left(A_{w 1}(h)-A_{w 2}(h)\right)^{2}+\left(A_{w 2}(h)-A_{w 0}(h)\right)^{2} \\
& =\frac{4}{25}\left[\left(h\left(F_{w} q_{0}\right)-h\left(F_{w} q_{1}\right)\right)^{2}+\left(h\left(F_{w} q_{1}\right)-h\left(F_{w} q_{2}\right)\right)^{2}\right. \\
& \left.\quad+\left(h\left(F_{w} q_{2}\right)-h\left(F_{w} q_{0}\right)\right)^{2}\right] .
\end{aligned}
$$


When we sum over all words $w$ of length $m-1$ we obtain exactly $\frac{4}{25} E_{m-1}(h$, $h)$, since every pair of adjacent vertices $(x, y)$ in $V_{m-1}$ occurs once and only once, namely with the unique word $w$ of length $m-1$ such that $x, y \in F_{w} K$. This gives the first term on the right side of (3.3) (when $m=1$ there are no other terms).

The second type of adjacent pair is of the form $\left(w j, w^{\prime} k\right)$ where $\left(w, w^{\prime}\right)$ is an adjacent pair in $\widetilde{\Gamma}_{m-1}$, and $F_{w} q_{j}=F_{w^{\prime}} q_{k}$. From (3.4) we see $A_{w j}(h)-$ $A_{w^{\prime} k}(h)=\frac{3}{5}\left(A_{w}(h)-A_{w^{\prime}}(h)\right)$. If we square and take the sum over all such pairs, we obtain exactly $\frac{9}{25} \widetilde{E}_{m-1}(h, h)$, completing the proof of (3.3).

We can also prove directly that $\widetilde{\mathcal{E}}_{m}(h, h)$ is independent of $m$. Let $\widetilde{E}_{m}^{0}(h, h)$ denote the contribution to $\widetilde{E}_{m}(h, h)$ from the first type of adjacent pairs. Then we can show

$$
\left\{\begin{array}{l}
\widetilde{E}_{m}^{0}(h, h)=\frac{3}{5} \widetilde{E}_{m-1}(h, h) \\
\widetilde{E}_{m}(h, h)=\widetilde{E}_{m}^{0}(h, h)+\frac{9}{25} \widetilde{E}_{m-1}(h, h),
\end{array}\right.
$$

and from this deduce that $\widetilde{\mathcal{E}}_{m}(h, h)$ is independent of $m$.

It is easy to see that the average values $a_{w}=A_{w}(h)$ of a harmonic function minimize the energy

$$
\sum_{w \sim m}\left(a_{w}-a_{w^{\prime}}\right)^{2}
$$

among all choices with the same boundary values $a_{j^{m}}$. However, if we fix the values of $a_{w}$ for $|w|=m-1$ and minimize the energy (3.6) subject to the consistency conditions (2.10), we will not obtain harmonic functions, even if (2.11) holds for $|w|=m-1$. It seems likely that the solution of this minimum extension problem is not local (the formula for $a_{w}$ would involve the values of $a_{w^{\prime}}$ for all words $w^{\prime}$ of length $m-1$, not just those in a neighborhood of $w$ ). We have worked out the solution for $m=2$, namely $a_{j k}=a_{j}+\frac{1}{4} a_{k}-\frac{1}{4} a_{\phi}$, which is quite different from (2.12), and this yields the relationship $\widetilde{E}_{2}=\frac{3}{4} \widetilde{E}_{1}$.

Because of this situation, the sequence $\widetilde{\mathcal{E}}_{m}(u, u)$ will not necessarily be monotone increasing, so we cannot obtain the exact analog of Proposition 2.1. But we can circumvent this difficulty by first considering piecewise harmonic functions. Let $\mathcal{H}_{m}$ denote the space of continuous functions whose restrictions to each $F_{w} K$, for $|w|=m$, are harmonic (meaning $u \circ F_{w}$ is harmonic). It is shown in $[\mathbf{K i 2}]$ that $\mathcal{H}=\bigcup_{m} \mathcal{H}_{m}$ is dense in dom $\mathcal{E}$, so knowing the behavior of $\widetilde{\mathcal{E}}_{m}(u, u)$ for $u \in \mathcal{H}$ will be very useful. 
Corollary 3.2. For $u \in \mathcal{H}$,

$$
\lim _{k \rightarrow \infty} \widetilde{\mathcal{E}}_{k}(u, u)=\mathcal{E}(u, u) .
$$

Proof. For simplicity of notation we prove this for $u \in \mathcal{H}_{1}$. In the computation of $\widetilde{E}_{m}(u, u)$ we consider two types of adjacent pairs (note these are not the same as the two types in the proof of Theorem 3.1). The first type are of the form $\left(0 w, 0 w^{\prime}\right),\left(1 w, 1 w^{\prime}\right)$ or $\left(2 w, 2 w^{\prime}\right)$ where $w \sim_{m-1} w^{\prime}$, and these contribute exactly

$$
\sum_{j=0}^{2} \widetilde{E}_{m-1}\left(u \circ F_{j}, u \circ F_{j}\right) .
$$

The second type consists of just the 3 pairs $\left(10^{m-1}, 01^{m-1}\right),\left(12^{m-1}, 21^{m-1}\right)$ and $\left(20^{m-1}, 02^{m-1}\right)$ that lie at the intersection points of $F_{j} K$ and $F_{k} K$. At these intersection points it is easy to bound the differences $a_{w}-a_{w^{\prime}}$ by a multiple of $(3 / 5)^{m}$, so the contribution to $\widetilde{E}_{m}(u, u)$ is bounded by a multiple of $(3 / 5)^{2 m}$. When we take into account the normalization factor in (3.2), these terms will vanish in the limit. Thus

$$
\begin{aligned}
\lim _{m \rightarrow \infty} \widetilde{\mathcal{E}}_{m}(u, u) & =\lim _{m \rightarrow \infty} \frac{3}{2}\left(\left(\frac{3}{5}\right)^{m}-\left(\frac{3}{5}\right)^{2 m}\right)^{-1} \sum_{j=0}^{2} \widetilde{E}_{m-1}\left(u \circ F_{j}, u \circ F_{j}\right) \\
& =\frac{5}{3} \lim _{m \rightarrow \infty} \sum_{j=0}^{2} \widetilde{\mathcal{E}}_{m-1}\left(u \circ F_{j}, u \circ F_{j}\right) \\
& =\frac{5}{3} \sum_{j=0}^{2} \mathcal{E}\left(u \circ F_{j}, u \circ F_{j}\right) \\
& =\mathcal{E}(u, u)
\end{aligned}
$$

by Theorem 3.1 .

It does not follow immediately from the density of $\mathcal{H}$ in $\operatorname{dom} \mathcal{E}$ that (3.7) continues to hold for all $u$ in dom $\mathcal{E}$, since this involves interchanging limits. The required maximal estimate is provided in the next Lemma.

Lemma 3.3. Let $u \in \operatorname{dom} \mathcal{E}$. Then

$$
\begin{gathered}
\left|u(x)-A_{w}(u)\right| \leq c \mathcal{E}\left(u \circ F_{w}, u \circ F_{w}\right)^{1 / 2} \quad \text { for all } \quad x \in F_{w} K, \\
\widetilde{\mathcal{E}}_{m}(u, u)^{1 / 2} \leq c\left(\mathcal{E}_{m}(u, u)^{1 / 2}+\mathcal{E}(u, u)^{1 / 2}\right),
\end{gathered}
$$

and

$$
\sup _{m} \widetilde{\mathcal{E}}_{m}(u, u) \leq c \mathcal{E}(u, u)
$$


Proof. It is shown in $[\mathbf{K i 2}]$ that $|u(x)-u(y)| \leq c \mathcal{E}(u, u)^{1 / 2}$ for any $x, y \in$ $K$, and applying this estimate to $u \circ F_{w}$ and averaging with respect to $y$ yields (3.8). To prove (3.9) we want to replace each term in $\widetilde{\mathcal{E}}_{m}(u, u)$ by a corresponding term in $\mathcal{E}_{m}(u, u)$, and use (3.8) to control the error. So for each pair of adjacent words $\left(w, w^{\prime}\right)$ of length $m$ we pick a pair of adjacent vertices $(x, y)$ in $V_{m}$ with $x \in F_{w} K$ and $y \in F_{w^{\prime}} K$. There are 4 different ways to make this choice, and depending on how the choice is made the same pair $(x, y)$ can be chosen twice (with some care we can eliminate duplicates, but it only affects the constant in the estimate). Writing

$$
A_{w}(u)-A_{w^{\prime}}(u)=u(x)-u(y)+\left(A_{w}(u)-u(x)\right)+\left(u(y)-A_{w^{\prime}}(u)\right)
$$

and making routine estimates we obtain

$$
\widetilde{E}_{m}(u, u)^{1 / 2} \leq c\left(E_{m}(u, u)^{1 / 2}+\left(\sum_{|w|=m}\left|A_{w}(u)-u(x)\right|^{2}\right)^{1 / 2}\right)
$$

where $x \in F_{w} K$. Now we use (3.8) to estimate this last sum. When we multiply by the renormalization constant in (3.2), which is of the order of $(5 / 3)^{m}$, we obtain

$$
\begin{aligned}
\widetilde{\mathcal{E}}_{m}(u, u)^{1 / 2} & \leq c\left(\mathcal{E}_{m}(u, u)^{1 / 2}+\left(\left(\frac{5}{3}\right)^{m} \sum_{|w|=m} \mathcal{E}\left(u \circ F_{w}, u \circ F_{w}\right)\right)^{1 / 2}\right) \\
& =c\left(\mathcal{E}_{m}(u, u)^{1 / 2}+\mathcal{E}(u, u)^{1 / 2}\right)
\end{aligned}
$$

by the self-similarity of the Dirichlet form $\mathcal{E}$. This proves (3.9), and since the constant is independent of $m$ and $\mathcal{E}_{m}(u, u) \leq \mathcal{E}(u, u)$ by Proposition 2.1, we have (3.10).

Theorem 3.4. If $u \in \operatorname{dom} \mathcal{E}$ then (3.7) holds. Conversely, if $u$ is continuous and

$$
\liminf _{k \rightarrow \infty} \widetilde{\mathcal{E}}_{k}(u, u)<\infty
$$

then $u \in \operatorname{dom} \mathcal{E}$.

Proof. Since $\mathcal{H}$ is dense in $\operatorname{dom} \mathcal{E}$ and (3.7) holds for $\mathcal{H}$, it follows by routine functional analysis arguments that it holds for $\operatorname{dom} \mathcal{E}$ because of (3.10).

Conversely, suppose $\widetilde{\mathcal{E}}_{m}(u, u) \leq M$ for an infinite number of indices $m$. We construct functions $u_{m}$ in $\mathcal{H}_{m}$ by setting

$$
u_{m}(x)=\frac{1}{2}\left(A_{w}(u)+A_{w^{\prime}}(u)\right)
$$

if $x \in F_{w} K \cap F_{w^{\prime}} K$, and $u_{m}\left(q_{j}\right)=A_{j^{m}}(u)$ on the boundary. Because $u$ is continuous, $u_{m}$ converges uniformly to $u$ as $m \rightarrow \infty$. On the other hand, 
we claim that $\mathcal{E}_{m}\left(u_{m}, u_{m}\right) \leq c \widetilde{\mathcal{E}}_{m}(u, u)$. The reason is that if $x \sim_{m} y$, then $x \in F_{w} K \cap F_{w^{\prime}} K$ and $y \in F_{w^{\prime}} K \cap F_{w^{\prime \prime}} K$, with $w \sim_{m} w^{\prime} \sim_{m} w^{\prime \prime}$, so

$$
u_{m}(x)-u_{m}(y)=\frac{1}{2}\left(A_{w}(u)-A_{w^{\prime}}(u)\right)+\frac{1}{2}\left(A_{w^{\prime}}(u)-A_{w^{\prime \prime}}(u)\right),
$$

and each neighboring pair of words arises at most 4 times in this way (a slightly different argument is needed if one of the vertices is a boundary point). Thus we have $\mathcal{E}_{m}\left(u_{m}, u_{m}\right) \leq c M$, but $\mathcal{E}_{m}\left(u_{m}, u_{m}\right)=\mathcal{E}\left(u_{m}, u_{m}\right)$ because $u_{m} \in \mathcal{H}_{m}$, so

$$
\mathcal{E}_{k}\left(u_{m}, u_{m}\right) \leq \mathcal{E}\left(u_{m}, u_{m}\right) \leq c M
$$

for all $k$ because $\mathcal{E}_{k}\left(u_{m}, u_{m}\right)$ is increasing in $k$. Now if we fix $k$ and let $m \rightarrow \infty$ we obtain

$$
\mathcal{E}_{k}(u, u) \leq c M
$$

since $u_{m} \rightarrow u$ uniformly. Since this is true for all $k, u \in \operatorname{dom} \mathcal{E}$.

\section{The Laplacian.}

In this section we describe how to obtain the Laplacian directly in terms of average values. In a sense this is unnecessary, since the Laplacian can be defined entirely in terms of the Dirichlet form, by $u \in \operatorname{dom} \Delta$ and $\Delta u=f$ if and only if $u \in \operatorname{dom} \mathcal{E}, f$ is continuous, and

$$
\mathcal{E}(u, v)=-\int f v d \mu
$$

for every $v \in \operatorname{dom} \mathcal{E}$ vanishing on the boundary. However, there is a much simpler and appealing definition in terms of pointwise difference quotients. Let

$$
\Delta_{m} u(x)=\frac{3}{2} 5^{m}\left(\sum_{y \sim_{m} x} u(y)-4 u(x)\right)
$$

for $x \in V_{m} \backslash V_{0}$. Then $u \in \operatorname{dom} \Delta$ and $\Delta u=f$ if and only if $u$ and $f$ are continuous and $\Delta_{m} u$ converges uniformly to $f$. (The renormalization factor $5^{m}$ is in fact the ratio $\left(\frac{5}{3}\right)^{m} /\left(\frac{1}{3}\right)^{m}$ where $\left(\frac{5}{3}\right)^{m}$ is the Dirichlet form renormalization factor and $\left(\frac{1}{3}\right)^{m}$ is the measure of $F_{w} K$ for $|w|=m$. The constant $3 / 2$ is needed to have (4.1) hold.)

Our goal is to replace $\Delta_{m} u$ by an analogous expression in terms of average values, namely

$$
\widetilde{\Delta}_{m} u(x)=\frac{3}{2} 5^{m}\left(\sum_{w^{\prime} \sim_{m} w} A_{w^{\prime}}(u)-3 A_{w}(u)\right) \quad \text { for } \quad x \in F_{w} K,|w|=m .
$$


Note that $\widetilde{\Delta}_{m} u$ is not defined on the boundary cells $F_{j^{m}} K$, just as $\Delta u$ is not defined at boundary points, but in the limit this is not important $(\Delta u(x)$ is defined at boundary points through the continuity of $f$ ).

Theorem 4.1. If $u \in \operatorname{dom} \Delta$ with $\Delta u=f$, then $\widetilde{\Delta}_{m} u$ converges uniformly to $f$.

Proof. Let $v_{0}$ denote the unique solution of $\Delta u=1$ that vanishes at the boundary. It is not hard to show that $\partial_{n} v_{0}\left(q_{j}\right)=-1 / 3$ for all $j$, so

$$
\int v_{0} \Delta u d \mu=\int u d \mu-\frac{1}{3} \sum_{j=0}^{2} u\left(q_{j}\right)
$$

by the Gauss-Green formula [Ki2]. Applying (4.4) to $u \circ F_{w}$ and using the scaling identity $\Delta\left(u \circ F_{w}\right)=5^{-m}(\Delta u) \circ F_{w}$ (see [Ki1]), we obtain

$$
A_{w}(u)-\frac{1}{3} \sum_{j=0}^{2} u\left(F_{w} q_{j}\right)=5^{-m} \int v_{0}(\Delta u) \circ F_{w} d \mu .
$$

We use (4.5) to compare $\widetilde{\Delta}_{m} u$ with $\Delta_{m} u$ and control the error. To do this we refer to Figure 2.3. The weights shown are arrived at in two different ways. The first way is to take

$$
\sum_{w^{\prime} \sim_{m} w} A_{w^{\prime}}(u)-3 A_{w}(u)
$$

and replace $A_{w}(u)$ by its approximation

$$
\frac{1}{3} \sum_{j=0}^{2} u\left(F_{w} q_{j}\right)
$$

and similarly for $A_{w^{\prime}}(u)$. The second way is to take

$$
\frac{1}{3} \sum_{x=F_{w} q_{j}}\left(\sum_{y \sim_{m} x} u(y)-4 u(x)\right) .
$$

(The weights just give the factor multiplying the value of $u$ at the corresponding vertex.) Since the two methods yield the same expression, we can obtain an identity with remainder by using the exact formula (4.5), namely (for $x \in F_{w} K$ )

$$
\widetilde{\Delta}_{m} u(x)=\frac{1}{3} \sum_{j=0}^{2} \Delta_{m} u\left(F_{w} q_{j}\right)+R_{m}(x)
$$


with

$$
R_{m}(x)=\frac{3}{2} \int v_{0}\left(3(\Delta u) \circ F_{w}-\sum_{w^{\prime} \sim_{m} w}(\Delta u) \circ F_{w^{\prime}}\right) d \mu .
$$

Since $\Delta_{m} u \rightarrow f$ uniformly, it follows from (4.6) that $\widetilde{\Delta}_{m} u \rightarrow f$ uniformly if we can show that $R_{m}(x) \rightarrow 0$ uniformly. But this is evident from (4.7) because $v_{0}$ and $\Delta u$ are continuous functions.

Theorem 4.2. If $u$ is a continuous (or even just integrable) function, and $\widetilde{\Delta}_{m} u$ converges uniformly to a continuous function $f$, then $u \in \operatorname{dom} \Delta$ and $\Delta u=f$.

Proof. We use the following criterion (weak implies strong) for $u \in \operatorname{dom} \Delta$ with $\Delta u=f$ :

$$
\int u \Delta v d \mu=\int f v d \mu
$$

for all $v \in \operatorname{dom} \Delta$ vanishing in a neighborhood of the boundary ([S1] and $[\mathbf{S U}])$. To verify (4.8) we observe directly from the definitions that

$$
\begin{aligned}
\widetilde{E}_{m}(u, v)= & \sum_{w \sim_{m} w^{\prime}}\left(A_{w}(u)-A_{w^{\prime}}(u)\right)\left(A_{w}(v)-A_{w^{\prime}}(v)\right) \\
= & -\sum_{w}{ }^{\prime} A_{w}(v)\left(\sum_{w^{\prime} \sim_{m} w} A_{w^{\prime}}(u)-3 A_{w}(u)\right) \\
& -\sum_{w}{ }^{\prime \prime} A_{w}(v)\left(\sum_{w^{\prime} \sim_{m} w} A_{w^{\prime}}(u)-2 A_{w}(u)\right)
\end{aligned}
$$

where the first sum $\sum$ ' extends over all $w$ not on the boundary, and the second sum $\sum$ " extends over the 3 boundary words $j^{m}$. Note that

$$
\frac{3}{2}\left(\frac{5}{3}\right)^{m} \sum_{w}{ }^{\prime} A_{w}(v)\left(\sum_{w^{\prime} \sim_{m} w} A_{w^{\prime}}(u)-3 A_{w}(u)\right)=\int v \widetilde{\Delta}_{m} u d \mu
$$

because $3^{-m} A_{w}(v)=\int_{F_{w} K} v d \mu$ and $\widetilde{\Delta}_{m} u$ is constant on $F_{w} K$ (by convention we exclude the boundary cells from the integral since $\widetilde{\Delta}_{m} u$ is undefined there). In (4.9) we can also interchange the role of $u$ and $v$. In both cases the boundary sum will vanish if $m$ is large enough because we are assuming that $v$ vanishes in a neighborhood of the boundary. Thus we obtain

$$
\int v \widetilde{\Delta}_{m} u d \mu=\int u \widetilde{\Delta}_{m} v d \mu \text {. }
$$


Now let $m \rightarrow \infty$. By assumption $\widetilde{\Delta}_{m} u \rightarrow f$ uniformly, and by Theorem 4.1 $\widetilde{\Delta}_{m} v \rightarrow v$ uniformly. This yields (4.8), even if $u$ is only assumed to be integrable.

It is also possible to characterize other kinds of weak solutions of $\Delta u=f$ (for example $f \in L^{2}$ ) in terms of $\widetilde{\Delta}_{m} u$, but we will not do this here.

\section{References}

$[\mathrm{J}] \quad$ A. Jonsson, Brownian motion on fractals and function spaces, Math. Zeit., 222 (1996), 495-504, MR 97e:60137, Zbl 863.60079.

[Ki1] J. Kigami, A harmonic calculus on the Sierpinski spaces, Japan. J. Appl. Math., 8 (1989), 259-290, MR 91g:31005, Zbl 686.31003.

[Ki2] _ Harmonic calculus on p.c.f. self-similar sets, Trans. Amer. Math. Soc., 335 (1993), 721-755, MR 93d:39008, Zbl 773.31009.

[Ki3] _ _ Analysis on Fractals, Cambridge Univ. Press, Cambridge, 2001.

[KZ] S. Kusuoka and X.Y. Zhou, Dirichlet forms on fractals: Poincaré constant and resistance, Probab. Theory Related Fields, 93 (1992), 169-196, MR 94e:60069, Zbl 767.60076.

[S1] R. Strichartz, Some properties of Laplacians on fractals, J. Funct. Anal., 164 (1999), 181-208, MR 2000f:35032.

[S2] _ Taylor approximations on Sierpinski gasket type fractals, J. Funct. Anal., 174(1) (2000), 76-127, CMP 1761 364, Zbl 956.31007.

[SU] R.S. Strichartz and M. Usher, Splines on fractals, Math. Proc. Camb. Phil. Soc., 129(2) (2000), 331-360, MR 2001c:28016.

[Z] L. Zalcman, Mean values and differential equations, Israel J. Math., 14 (1973), 339352, MR 49 \#613, Zbl 263.35013.

Received July 9, 1999 and revised January 5, 2000. This research was supported in part by the National Science Foundation, Grant DMS 9970337.

Mathematics Department

MaLott HaLL

CORNELl University

ITHACA, NY 14853

E-mail address: str@math.cornell.edu 